\title{
New standard for ALK-positive NSCLC
}

$A L K$ rearrangements are present in about $3-5 \%$ of patients with non-small-cell lung cancer (NSCLC). On the basis of the remarkable results of a phase I-II study that showed high tumour response rate and prolonged progression-free survival (PFS) with crizotinib in patients with $A L K$-positive lung cancer, the next logical step was to prove the efficacy of this agent as first-line therapy versus standard platinum-based chemotherapy in patients.

As part of a close collaboration between investigators and sponsors, Ben Solomon and Tony Mok, who coauthored this study, provide background to this latest phase III randomized trial: "this was a classic 'marker-based strategy design' that screened all patients with advancedstage NSCLC and enrolled only patients with the $A L K$ rearrangement," explains Mok. In total, 343 patients were randomly assigned to crizotinib or pemetrexed in combination with cisplatin or carboplatin chemotherapy. The primary end point was PFS and secondary end points included response rate, overall survival and quality of life. Crossover to crizotinib was permitted for patients with disease progression who received chemotherapy. PFS was significantly longer with crizotinib (10.9 months versus 7.0 months) and so was the objective response rate (74\% versus $45 \%$ ) compared with chemotherapy. Crizotinib was also associated with a better reduction in lung cancer symptoms and quality of life. Overall survival was not significantly different, likely owing to crossover.

"We have taken 'personalized medicine' for lung cancer to a higher level," says Mok, and Solomon further adds: "this trial establishes crizotinib as the standard-of-care in previously untreated patients whose tumours test positive for ALK rearrangements."

\section{Lisa Hutchinson}

Original article Solomon, B. J. et al. First-line crizotinib versus chemotherapy in ALK-positive lung cancer. N. Engl. J. Med. doi:10.1056/NEJMoa1408440 